\title{
Lessons learned from one decade of developing agent-based tools for traffic modeling, simulation, and control: how to make cities smarter
}

\author{
Ana L. C. Bazzan \\ ${ }^{1}$ Instituto de Informática - Universidade Federal do Rio Grande do Sul (UFRGS) \\ Caixa Postal 15.064 - 91.501-970 - Porto Alegre - RS - Brazil \\ bazzan@inf.ufrgs.br
}

\begin{abstract}
While there is no definitive agenda for making cities smarter in terms of mobility, intuition says that information is key. However, contrarily to a more technical view, information should not only be consumed by the users, but also produced by them. This is already happening (smart-phones, social networks) but is far from being exploited by technical systems related to transportation. In this paper I discuss some of the issues related to more human-centered transportation system. It is argued that users of this system are somehow influencing it with their increasing coupled behaviors. Thus, there is a real opportunity for changing the paradigm in order to take advantage of the collective intelligence.
\end{abstract}

\section{Introduction}

What makes a city smart? This question has no definitive answer. Most likely, it is a combination of factors and actions that may characterize a city as less or more smart. However, it is well accepted that to have smart cities, a combination of smart transportation/mobility, smart energy (and buildings), and smart fighting of crime (security issues) is key. The present paper deals with the former.

The aim here is to report a personal view and experience, greatly influenced by the cooperation with several colleagues, about how to mitigate traffic problems by means of human-centered modeling, simulation, and control. By human-centered it is meant that it is necessary to put the human in the loop of traffic control and decision-making. This may look obvious at first. However, such an endeavor is far from trivial. As discussed later, only recently, with the increase in I\&C technologies, it has turned possible (in fact, unavoidable) to have users of the traffic and transportation systems in urban areas as both targets (or objects) and as active subjects. This means that instead of only passively receiving information (when it is available at all) or passively waiting for the light to turn green, the user now has the possibility to interact with the system in various ways. The most important one is of course providing information, acting as a human sensor. Thus changing the user's role of actuator to sensor is a real change in paradigm.

In this sense, human-centered modeling, simulation, and control of traffic starts to be a reality. Only the former can really be said to be (partially) accomplished. This is due to notorious changes in the way modeling and simulation is done. The spreading usage of the microscopic and, in particular, agent-based simulation paradigm is making possible that individuals (or classes of them) that participate in the traffic system are modeled with their idiosyncrasies. Moreover, this also permits the modeling and simulation of time 
frames of days or even minutes (whereas macroscopic simulation deals with longer time frames, normally for planning purposes). Thus, human-centered simulation is on its way, but huge challenges are still there.

As for human-centered traffic control, it is less obvious that this may indeed ever happen. What is in fact a human-centered traffic control? It is certainly not related to actuated traffic control (i.e., a traffic light that is actuated upon the volume of cars, delays, queues, and so forth), even if in each car waiting in a queue, there is at least a human being (possibly unhappy with the situation). The idea of human-centered control relates in fact to a smarter control at network level, meaning a smarter distribution of flows, a smarter use of space, time, and transportation mode. This all requires a fundamental thing: data. This data is usually expensive to obtain (in most medium to large cities, transportation authorities are clueless about microscopic patterns, users preferences, and that like). The consequences are notorious: traffic authorities can, at most, react to traffic occurrences. They are however completely blind to predictive acting. Often, system users know better than the traffic authorities themselves about traffic patterns in routes that are frequently used by them. This knowledge however is traditionally not used. Thus smart cities also mean that the potential of the collective intelligence cannot be disregarded. This poses some challenges for information systems because data can be non accurate, conflicting, partial, non up-to-date, and especially, be produced in huge quantities.

Finally, paradoxically as it seems, maybe one way out of the mess is to take the human out of the driver's seat and have only autonomously driven cars for mobility purposes. Autonomous vehicles make sense not only by freeing the human driver to perform other activities while being driven, but also allow smaller gaps between vehicles, thus increasing up to $30 \%$ the roads capacity, while also reducing the emissions significantly.

All these measures (but not only these) can contribute to making cities smarter. This is overdue: The number of large metropolitan areas with more than ten million inhabitants is increasing rapidly, with the number of these so-called mega-cities now at more than 20. For comparison, in 1950 there were 83 cities with populations exceeding one million, with New York being the sole city with population above ten million. This increase in the number of mega-cities has strong consequences to traffic and transportation. According to the keynote speaker of the IEEE 2011 forum on integrated sustainable transportation systems, Martin Wachs, mobility is perhaps the single greatest global force in the quest for equality of opportunity because it plays a role in offering improved access to other services. In fact, in the last decade, mobility patterns have changed drastically: it is no longer the case that congestion problems affect mainly US highways used by commuters, and European big cities. On the contrary: congestion is mentioned as one of the major problems in various parts of the world, leading to a significant decrease in the quality of life, especially in mega-cities of countries experiencing booming economies. According to www. its. dot.org, in 2010 there were 32,788 traffic-related deaths in the United States alone. Mobility is severely impacted with 4.8 billion hours of travel delay that put the cost of urban congestion at 114 billion dollars. Moreover there are still the costs to the environment (3.9 billion gallons of wasted fuel emissions).

In the next section, some aspects in terms of modeling, simulation, and control of traffic are discussed, including challenges ahead, with a bias towards how to contribute to the effort of having smarter cities. Section 3 summarizes and proposes an agenda to 
tackle the challenges mentioned.

\section{Smart Modeling, Simulation, and Control of Traffic: focusing on the human}

A transportation system consists of two main components: supply and demand. The former is made up of the infrastructure such as roads, public transportation (transit), etc. (see Chapter 1 of [Cascetta 2009] for more details). Demand regards the mobility needs of a population. It results in trips that are made at given times from and to different locations, using given transportation modes. Of course both components are closely related. For one side, mobility patterns are influenced by the supply. Also, when the demand exceeds the capacity of the roads, congestion arises. This, on its turn, affects the travel choices.

These (and other) processes and phenomena that occur in a transportation system are complex, with various cycles, i.e., non-linear interactions. Thus, in order to design, change, or evaluate a transportation system, normally models of it (either as a whole or parts of it) need to be produced. Chapter 1 of [Cascetta 2009] is a good overview of the various issues underlying such a task. Due to lack of space, modeling and simulation will be discussed at a high level. At such level, both overlap so that they can be explained together. For the purpose here, one may however consider the following distinction: Modeling is the development of a model as a representative of a system. Simulation can be defined as experimenting or executing such a model.

In a traffic and transportation system, modeling and simulation normally is associated with one or more of the these three tasks: supply models, demand models, and assignment models. The latter is based on an interaction between demand and supply because its aim is to predict how trips between origins and destinations will use the existing infrastructure in a given time span.

Modeling and simulating supply aim at representing the performance of the available infrastructure, as well as factors such as emissions and accidents.

Modeling and simulating demand traditionally aims at predicting high-level aspects of demand for trips as a function of the available supply. This means that the aim here is towards long-range planning.

Related to the latter, modeling and simulation of assignment is normally also done for planning purposes. Traditionally, this process is an equilibrium-seeking one. As mentioned, we are concerned here with human-centered modeling. Thus, agent-based models of demand assignment have become more popular. The motivations for using them are manifold: increasing complexity of trips; heterogeneity can be represented; individual adaption and learning methods can be used; integration of interactions among agents; integration of other levels of decision-making such as mode choice; etc.

Modeling and, especially, simulation can then be used in several tasks: strategic and tactical planning, feasibility studies, and management of the operation of the system. While the planning and feasibility studies are important in designing and fixing smart cities, the prototypical application of modeling and simulation when it comes to smart transportation is the latter. Thus this is the focus next.

Given the current developments in communication and hardware, computer-based traffic control and management of the traffic system is now a reality. Nowadays the term 
"advanced transportation management system" (ATMS) is used to denote the set of all control and management technologies. The main goals of an ATMS are: to maximize the overall capacity of the network; to maximize the capacity of critical routes and intersections which represent bottlenecks; to minimize the negative impacts of traffic on the environment and on energy consumption; to minimize travel times; and to increase traffic safety. Besides, modern philosophies of ATMS also attempt to efficiently manage the communication between driver, vehicle, and roadway components (e.g. traffic signals).

There are several concepts of computer-based ATMSs. For details, the reader may refer to [Bazzan 2007]. For the sake of this discussion, what is worth mentioning is that conventional concepts are doomed to fail to tackle unexpected situations. However, these are those that cause the major damage. Just think about strong rain and flooding in major southeast Brazilian cities. Or the recent event of a new church attracting hundreds of thousands of people in the Guarulhos airport area, when a number of passengers lost their flights due to the complete blocking of the approaching roads. Despite thousands of tweets by desperate passengers, authorities have failed to cope with the situation, both at predictive as well as a reactive levels. Now imagine what would have been different if the operational center in charge of the highway, or of the airport, or of the municipality of Guarulhos, would have had prompt access to this huge amount of human sensors (here by means of people's smart phones).

Exactly because such connection is virtually inexistent, reported benefits on ATMSs virtually always refer to more or less normal situations (e.g. minor blockage of some roads, accidents, etc.). Unfortunately ATMSs were devised with the traffic authority in mind, not the citizen. Thus data collection and processing tend to be at high technical level, over a very sparse (because expensive) network of sensors (cameras, loop detectors). The only connection between citizens and ATMSs happens when the latter is used to generate information to the former, a practice that underlies the so-called "advanced traveler information system" (ATIS). An ATIS aims at providing information to travelers of both highway and urban systems. Information about the transportation network before and during travel is transmitted to the traveler using infrastructure-provided equipment as well as personal assistants and vehicle-based devices. En-route and pre-trip driver information, route guidance, and emergency notifications are examples of ATIS. ATIS can reduce travel times by giving users the information needed to select the most appropriate route, mode, or departure time for a given trip. Traveler information is particularly beneficial in situations when travelers are unaware of travel conditions on the highway network. These conditions could include incidents or bad weather.

One of the challenges of ATIS is to be integrated with an adequate modeling of assignment and with the management of the operations. This becomes more and more important for dynamic route guidance, for instance. Not only this: ATISs must turn into two-way, interactive systems where the user may also act as a provider of information (i.e., not only a target of it). In fact, the information gathering (from the users) is likely to end up being more important than the information provision (to the users), as it helps to understand travel behaviors. It is well-known that the response of users to information is still an open question (e.g., [Ben-Akiva et al. 1991, Bonsall 1992]). Moreover it is not clear whether more information is beneficial [Ben-Akiva et al. 1991]. Drivers confronted with too much information may become overloaded in the sense that information pro- 
cessing turns too difficult and users develop simple heuristics to solve the problem. These heuristics may lead to overreaction by the drivers, with non-expected effects. In the city of S. Paulo, on the eve of a holiday, it was recommended that users should plan their trips to Santos for the late hours of the night. This has caused immense jams around 3 a.m. because people overreact to the recommendation and decided to postpone their trips. This way, it is necessary to plan the type and frequency of the recommendations, and try to anticipate the effects of the information broadcast. If possible, the behavior of the drivers has to be incorporated in the forecast (e.g. [Ben-Akiva et al. 1991, Bonsall 1992]).

In order to generate a recommendation or an advice, data must be gathered in quantities that exceed the currently available one. Thus alternatives are necessary. Here a first possibility is to use data collected from taxis equipped with GPS ${ }^{1}$, smart-phones, and even social networks. In the future however, cars will be sold with several automated abilities, such as V2V communication. Thus this opens up further possibilities for data gathering that are already being investigated.

Finally, a further scenario where ATMSs and ATISs can cooperate and interact with the user is road pricing. This has been discussed several times for the metropolitan area of S. Paulo, but never really put forward. Admittedly, tolls are not popular. However, variants of it, in which the user may end up receiving money or credits, haven been also proposed. In the congestion toll variant, a toll is computed which is the difference between the marginal social cost and the marginal private cost. Notice that this difference can be negative meaning that some users actually get a reimbursement. This mechanism is not like toll charging for the sake of covering costs of road maintenance or simply for profit.

In summary, both ATMSs and ATISs must change their way of working in order to take advantage of the huge potential of having human sensors forming a kind of collective intelligence. The challenges here however, are non-neglectful. First of all, only inter-disciplinary approaches will be able to cope with this endeavor. Thus, researchers and practitioners on AI, database, data mining, information systems, distributed systems, middleware, data network, embedded systems, and computer and electronic engineering must address the following issues: Development of large scale agent-based modeling and simulation of millions of individuals; Information gathering from millions of sensors, cameras, and GPS; Data storage, maintenance and integration (GIS etc.); Broadcast via mobile devices (interfaces, etc.); Interoperability of on-board, on-route and control devices; Decentralized coordination of conventional traffic lights; Mechanisms to implement congestion tolls; Automated vehicles and intersections.

Above all, such a change of paradigm underlying the functioning of ATMSs and ATISs is likely to have behavioral and sociological implications. Just to mention one, navigation devices such as those based on GPS are becoming ubiquitious. Although we all seem to enjoy this help, little has been investigated about the effects of this use, especially on the travelers whose routes were mostly restricted to local streets, while the majority of other travelers would use basically arterials (and secondary links close to their origins and destinations), which are part of their respective mental maps. Two questions then arise. The first is whether travel time decreases if navigation devices get widely used. The second question relates to the effects of the use of these devices on travelers who use the

\footnotetext{
${ }^{1}$ In order to estimate the benefits for Brazilian cities, one must only think that the taxi fleet of S. Paulo for instance is over $30 \mathrm{~K}$ vehicles (source: CET, December of 2006).
} 
network only for short trips in their own neighborhood, thus tending to avoid arterials. Is it, as expected, that the flows tend to be more evenly distributed (as the navigation device can consider routes that a driver would never try), thus leading to lesser travel times? But then, what happens with the previous users of local streets? How will they react to a major increase in their travel times? This is investigated in [Bazzan and Azzi 2012], where the driving of thousands of agents is simulated, which compute their own route based either on global knowledge of the traffic network, coming from navigation devices, or based on their mental maps of the network, which are necessarily partial.

\section{Conclusion}

In summary, in this paper I have outlined some of the issues related to bringing the human user closer to a, by now, very technical system which is the transportation system. It is argued that users of this system (virtually anyone!) are somehow influencing it with their increasing coupled behaviors. This is happening in spite of the will of the managers of these systems. Thus, it is a real opportunity for these to change the management paradigm in order to take advantage of the collective intelligence that is present in the real-world system (i.e., beyond the existing models).

In a short paper as the present one, it is quite impossible to propose and discuss a methodology to tackle such a complex problem. Therefore I can at most propose an agenda to address these and possibly other issues:

1. set priorities regarding the main issues to be tackled (re-design of parts of the transportation system? focus on control and operation management? focus on smart citizens?)

2. constitute inter-disciplinary working groups to come out with a list of requirements for smart transportation and traffic in smart cities;

3. once the requirements are established, define the set of actions;

4. involve the citizen in all stages of decision-making.

\section{Acknowledgments}

The authors wish to thank CNPq (for the support to the project LabTrans) and FAPERGS (for the support to the RS-SOC project).

\section{References}

Bazzan, A. L. C. (2007). "Traffic as a complex system: Four challenges for computer science and engineering”. In Wagner, F. R., Granville, L. Z., and Ishikawa, E., editors, Proc. of the XXXIV SEMISH, pages 2128-2142. SBC.

Bazzan, A. L. C. and Azzi, G. G. (2012). "An investigation on the use of navigation devices in smart transportation systems". In Anais do VIII Simpósio Brasileiro de Sistemas de Informação. SBC.

Ben-Akiva, M., de Palma, A., and Kaysi, I. (1991). Dynamic network models and driver information systems. Transp. Res. A, 25(5):251-266.

Bonsall, P. W. (1992). The influence of route guidance advice on route choice in urban networks. Transportation, 19(1).

Cascetta, E. (2009). Transportation Systems Analysis - Models and Applications, volume 29 of Springer Optimization and Its Applications. Springer. 